\title{
Road to Rome: Commercialization of Point-of-Care Devices and Academic Research
}

\author{
Chandra K Dixit* \\ Department of Chemistry, University of Connecticut, Storrs, CT 06269, USA
}

\begin{abstract}
Received: September 07, 2015; Accepted: September 28, 2015; Published: October 05, 2015
*Corresponding author: Chandra K Dixit, Department of Chemistry, University of Connecticut, Storrs, CT 06269, USA, Tel: +18602086000; E-Mail: chandrakumar.dixit@gmail.com
\end{abstract}

Point-of-Care (POC) Testing is also referred to as decentralized or bedside testing. According to the Clinical Laboratory Improvement Amendment (CLIA) criteria, POC testing is generally divided into waived and non-waived testing categories based on their operational complexity [1]. Any non-clinical test with an approval from the US Food and Drug Administration (FDA) which employs very simple and accurate methodologies such that probability of erroneous results is either negligible or presents no reasonable risk to the patients upon being used inappropriately is considered in CLIA-waived category. Such tests are enlisted on CDC website and are constantly being updated. The Nonwaived testing category includes moderately to highly complex tests which require certain degree of scientific and technical knowledge and training to perform them accurately. Operational steps in such tests are either easily controlled with certain degree of automation or may have to be strictly controlled with appropriate monitoring. Extensive analytical interpretations and judgements made by highly trained and qualified personnel is the key to CLIA non-waived category.

Currently, developing POC testing for cancer has several restrictions. The foremost of them is integrating several tests together on a single device such that no single test interferes with the performance of the other. In addition, this must be achieved while allowing inexperienced people to perform such tests and enabling them to understand and analyze the results without the need of an expert. Therefore, the next generation Companion Diagnostics (CDx) and POC development will be governed by the demands of target users in resource-limited settings. In addition to the manufacturing, stringent FDA and CLIA approvals pose major restriction for rapid development of POC tools. On the manufacturing front, rapid prototyping of essential POC components, such as valves, mixers, pumps, and microfluidic chips, allow for optimizing designs with minimal costs $[2,3]$. However, packaging all the individual components into a functional POC increases the overall cost drastically.
Given all these restrictions in the POC development, only few products originating out of academic research reach to the market. This scenario seems to change due to increased industryacademia collaborations. As a result, we started to see spin-offs originating from, within the academic settings having products aimed for CDx. In the USA, there are 156 spin-offs registered in the field of disease diagnostics since 2008 while only 17 are from academic institutes and universities. These numbers increased from 1 in 2008 to 3 in 2015 [4]. This data is suggestive of majority of the academic research in this field never gets translated. To overcome this, in my opinion the best strategy could be to begin designing the devices and operations as per the guidelines of FDA and CLIA $[5,6]$. This may increase the translational output and we may see increased number of commercial products and spin-offs.

\section{References}

1. Centers for Medicare and Medicaid Services. Clinical Laboratory Improvement Amendments (CLIA). 2015.

2. Akhmetov I, Ramaswamy R, Akhmetov I, Thimmaraju PK. Market Access Advancements and Challenges in "Drug-Companion Diagnostic Test" Co-Development in Europe. J Pers Med. 2015; 5(2): 213-28. doi: 10.3390/jpm5020213.

3. Dixit CK, Kadimisetty K, Otieno BA, Tang C, Malla S, Krause CE, et al. Approaches to Low Cost, High Sensitivity, Automated, Multiplexed Protein Immunoassays for Cancer Diagnostics. Analyst. 2015; DOI: 10.1039/C5AN01829C.

4. US Food and Drug Administration. List of Cleared or Approved Companion Diagnostic Devices (In Vitro and Imaging Tools). 2015.

5. Kaushik A, Tiwari S, Dev Jayant R, Marty A, Nair M. Towards detection and diagnosis of Ebola virus disease at point-of-care. Biosens Bioelectron. 2016 Jan 15;75:254-72. doi: 10.1016/j.bios.2015.08.040.

6. Vashist SK, O’Sullivan SA, O’Neill FT, Holthofer H, O’Reilly B, Dixit CK. A multi-well plate for biological assays. WIPO Patent W02010044083. $2010 ; 24$. 